\title{
Effect of optical defocus on colour perception
}

\section{${ }^{*}$ RR Sehlapelo and *AO Oduntan}

\author{
Department of Optometry, University of Limpopo, Private Bag X1106, Sovenga, 0727 South Africa \\ *<alabio@ul.ac.za>
}

\begin{abstract}
Daily experience shows that colour of a very distant object cannot be accurately determined. It is assumed that visual acuity (VA) loss is one of the factors at play in this case. The effects of reduced VA as a result of refractive error or optical defocus on colour vision have not been examined. Such study will dictate the need or otherwise for optical correction before assessment of colour vision. The purpose of this study therefore, was to investigate the effects of optical defocus on colour vision in individuals with normal colour vision. Twenty nine young adult subjects (11 male and 18 females) were included in this study. Their ages ranged from 11 to 29 years with a mean of $22.1 \pm 3.4$ years. All subjects had VA of $6 / 6$ or better and normal colour vision. The colour vision was evaluated with the Farnsworth panel D-15 (desaturated). Each subject was optically defocused to VA of 6/24, 6/60 and 1/60 (6/360) at 6 meters respectively and colour vision was assessed at each reduced VA. At VA of 6/24, colour vision was not affected in all subjects. When the VA was reduced to 6/60, however, $15(51 \%)$ of the subjects failed the colour vision test. When the VA was further reduced to $1 / 60$ by optical defocus, $24(83 \%)$ of the subjects failed the colour vision test. It was concluded that optical defocus and reduced VA can adversely affect colour vision test findings. While VA reduction to $6 / 24$ may not affect the colour vision results, VA of 6/60 can. It is therefore, recommended that in clinical colour vision testing and colour vision screening, VA should first be assessed and compensated, especially if uncorrected VA is $6 / 24$ or worse.
\end{abstract}

Keywords: Colour vision, Farnswoth Panel D-15 test, Visual acuity, Optical defocus, Optical blur, Refractive error.

\section{Introduction}

Colour is an extremely important component of the information that we gather with our eyes and most of us use it so automatically that we fail to appreciate how important it is in our daily activities. It serves as a non-linguistic code that gives us instant information about the world around us ${ }^{1}$.

In the eye, there are two types of photoreceptors, namely rods and cones. Rods mediate vision at low light levels and thus serve vision only under conditions such as at night; in contrast,cone photoreceptors mediate vision under light levels ${ }^{1}$. Primates including man are trichromats; that is they all have three types of cones that respond selectively to short (S), medium (M) and long (L) wavelengths of visible light, a narrow band of electromagnetic spectrum, wavelength between 400 and $700 \mathrm{~nm}^{2}$ Spectral sensitivity studies of cone photopigments have identified blue green and red cone photoreceptors. A minimal requirement for colour discrimination is the presence of at least two kinds of cone pigments (dichromats), but normal colour vision requires the presence of all the three trichomats ${ }^{3}$.

The content of this article was presented by Professor Oduntan at the Mopane Visual Optics Conference, Kruger National Park, Limpopo Province, South Africa, 06-09 August 2006.

*BOptom

* Bsc (Hons) Optom PhD

Received 22 January 2007; revised version accepted 9 July 2007 
In humans colour vision can be normal or impaired. Normal colour vision is trichromatic, meaning that all the hues can be matched by an additive mixture of three primary colours, taken from the long wavelength, 'red'; medium wavelength; 'green' and short wavelength 'blue' regions of the spectrum ${ }^{4}$. Defective colour vision is commonly called "colour blindness"and may arise from defects in any of the three cones (Iregren). Defective colour vision is characterized by abnormal colour matching and colour confusions. There is also a significant reduction in the number of spectral hues which can be distinguished and alterations in the spectral curve of relative luminous efficiency ${ }^{4}$.

Colour blindness can be hereditary (genetic) or acquired. The most common type is the genetic photoreceptor disorders. Congenital colour deficiency or dyschromatopsia arises from abnormal visual pigment photochemistry. The retina may be lacking in functional cone receptors or there may be only one or two photopigments in the cones, instead of three. Although three photopigments may be present, one of them may have abnormal absorption characteristics ${ }^{4}$. Acquired colour deficiency may ensue through damage to the retina, optic nerve, or higher brain areas. Higher brain areas implicated in colour processing include the parvocellular pathway of the lateral geniculate nucleus of the thalamus, and visual areas V4 of the visual cortex ${ }^{1}$. Acquired colour deficiency is associated with many blinding disorders, such as glaucoma, diabetic retinopathy, macular degeneration and exposure to certain toxic drugs and chemicals ${ }^{1,5}$.

In a recent review article, Rubin ${ }^{6}$ presented a detailed review of fundamental aspects of colour perception which included the sensory mechanism, molecular aspects for awareness and the human colour pathway. Also, the various anomalies of dichromacy and trichromacy have been described in many textbooks of optometry and ophthalmology, hence details of these aspects will not be included in this introduction.

Colour perception is one of the visual functions that are often measured clinically to identify and differentiate congenital and acquired colour deficiency and to select personnel for occupations which require good colour vision. Abnormal colour vision has limiting implications for many vocations where colour judgments have to be made. Obviously, a job that requires accurate matching of colours, for example in the dye and textile industries would be unsuitable for an individual with even mild colour discrimination problems ${ }^{7}$. Likewise, in the electrical and electronic industries, the ability to identify the colour coding of cables, wires, resistors and capacitors in different ambient illumination conditions is required ${ }^{7}$. Colour defective drivers have serious difficulties with colours of traffic signals, and break lights ${ }^{8}$. People who are colour vision deficient may be excluded from occupations such as train driving where there is a well accepted safety risk. Colour vision standards are imposed on the road, rail, maritime and aviation industries and are often subject of vigorous opposition by those excluded by the standard ${ }^{9}$. Many teaching materials for reading and mathematics are colour-coded in the pre-school, hence, children with colour vision impairment may have difficulty in their early education ${ }^{10}$.

Changes in colour vision can precede alteration in other visual functions such as acuity and visual fields and can thus serve as early warning to the presence of diseases ${ }^{10}$. Therefore, colour vision testing is an integral part of optometric services. Clinical colour vision tests are simplified versions of psychophysical methods and are based on pigment colours instead of spectral stimuli ${ }^{11}$. Hue discrimination tests are grading tests which identify moderate and severe colour deficiency and classify protan, deutan and tritan defects. The Farnsworth panel D15 is one of the most widely used hue discrimination test. The individual colours are included as circular caps which subtend $1.5^{\circ}$ at a test distance of $50 \mathrm{~cm}$, ensuring that observations are made with the central rod-free retinal area. ${ }^{11}$ The Lanthony (desaturated) panel D-15 can be used to assess fine colour discrimination, however, it has considerable within-subject variability in test results ${ }^{12}$.

Daily experience shows that it is difficult to accurately discern the exact colour of very distant objects such as the colour of a house, or car or leaves of a tree. Reduced visual acuity due to long distance may be one of the factors which contribute to this degradation of colour of distant objects. If this assumption is correct, the question that follows is: Will reduced visual acuity (VA) such as in case of uncompensated refractive error influence clinical colour vision findings? Sometimes textbooks of optometry ${ }^{13,14}$ do not clearly indicate whether or not refractive error should be compensated for before the colour vision testing.

A previous study ${ }^{15}$ has examined the effect of visual acuity on colour vision in relation to amblyopic patients. The authors ${ }^{15}$ concluded that colour vision in amblyopic eyes was not related to poor visual acuity. 
Although the effects of simulated visual impairment (dioptric blur) have been investigated clinically in relation to visual functions such as contrast sensitivity ${ }^{16,17}$, no study has been carried out on the effects of optical defocus (optical blur) clinically on colour perception of normally sighted subjects. Such study is necessary, as it will indicate the need or otherwise for ophthalmic compensation before colour vision tests are carried out in the clinic. The purpose of this study therefore, was to study the effect of optical defocus on the colour vision test findings.

\section{Method}

Subjects for this study were randomly selected among the University of Limpopo students. Criteria for inclusion included: no previous history of eye disease or injury. Visual acuity was measured at six meters for each subject using a Snellen acuity chart. Only those with uncompensated VA of 6/6 or better were included in the study. When VA was $6 / 6$ or better, the baseline colour vision was tested with the Farnsworth panel D15 (desaturated) from Bernell Corporation, USA. All caps, except the reference cap were removed from the box and mixed in front of the subject who was asked to arrange the caps into the box in a natural order, starting from the reference colour. The subject was given enough time to arrange the caps. When all the colours have been arranged, the subject was encouraged to look along the order of arrangement to see if changes were necessary. After this, the caps were turned upside down to check the order of numbers on the back of the caps. The numbers on the caps were recorded in the record sheet. A single error of two steps was regarded as a failure. In view of the possibility of within-subject variability in test results ${ }^{12}$, the test procedure was done twice. Those who failed the baseline colour vision test were excluded from further tests. Subsequently, the subject was optically defocused binocularly to VA of $6 / 24$ using positive lenses in a trial frame, and the colour testing was done twice and findings recorded. The subject was subsequently defocused further to visual acuity of $6 / 60$ and the testing was repeated twice and again recorded. Finally, the subject was further defocused to visual acuity of $1 / 60$ and again, testing was done twice and results were recorded in the recording sheet. The tests were conducted in a clinic room with daylight florescent. The room illumination was not measured because a suitable light meter was not available.

\section{Results}

The subjects included in the study were 29 and included 18 males and 11 females. They were all young adults, their ages ranged from 11 to 29 years, mean $=22.1(\mathrm{SD} \pm 3.4)$ years. The positive lens powers used to defocus the subjects to VA of $6 / 24,6 / 60$ and $1 / 60$ were: range, 2.00 to 6.25 , mean $=4.1 \mathrm{D}, \mathrm{SD}= \pm 1.2 \mathrm{D}$; range, 2.50 to $8.00 \mathrm{D}$ with a mean of $5.8 \mathrm{D}, \mathrm{SD}= \pm 1.6 \mathrm{D}$; range, $11.00 \mathrm{D}$ to $16.00 \mathrm{D}$, mean $=13.0 \mathrm{D}, \mathrm{SD}=$ $\pm 1.5 \mathrm{D}$ respectively. Following optical defocus to VA of $6 / 24$ at six meters, all the subjects passed the colour vision test but with optical defocus to VA of $6 / 60,15(51.7 \%)$ of the subjects failed the test. Following optical defocus to VA of 1/60, $25(86.2 \%)$ of the subjects failed the test.

\section{Discussion}

This study shows that optical defocus influences colour vision, although the VA at which this would occur was found to be worse than $6 / 24$. This suggests that uncompensated refractive error which degrades VA beyond $6 / 24$ can affect colour vision test findings. An interesting finding was that, after optical defocus to $6 / 60$, more than half $(53 \%)$ of the subjects failed the colour vision test. However, even when optical defocus reduced the VA to $1 / 60,4(13.8 \%)$ still passed the test. This suggests that there is a high intersubject variability in the way that colour perception is affected by optical defocus. This may be attributed to differences in sensitivity to optical defocus among the subjects. It may, however, be partly due to high intersubject variability in panel D-15 test as previously reported ${ }^{12}$.

After the completion of the study it was found that a laboratory study had just been published in the November 2005 issue of the Investigative Ophthalmology and Visual Science ${ }^{18}$ which investigated the effects of defocus, target size, ambient illumination and surround characteristics on the extent of the colour misperception of train signals by nine visually normal participants. The study demonstrated that small red targets (approximately $1 \mathrm{~min}$ arc) can appear yellow when viewed through small amounts of defocus of about $0.75 \mathrm{D}$ under bright illumination $\left(1910 \mathrm{~cd} / \mathrm{m}^{2}\right)$. The potential for this type of misconception to result in 
collision and fatalities was considered a major concern to the authors. Also, a similar clinical study was published by McCulley et al. ${ }^{19}$ in 2006. Using three test devices namely: Panel D15, Ishihara and HardyRand-Rittler (HRR), the authors defocused the subjects at a $0.1 \log$ MAR interval to 1.8 prior to colour vision assessment. They found that colour vision results did not differ significantly up to logMAR 1.4 for panel D-15, $\log$ MAR 1.10 for HRR and $\log$ MAR 0.72 for Ishihara plates respectively. (The Snellen equivalents of these values are approximately $6 / 85,6 / 65$ and 6/30 respectively.) The authors found that optical defocus (worse than 6/30 approximately) affects colour perception and that the devices differed significantly $(p<0.005)$ in the way that they are influenced by optical defocus.

The present findings agree with those studies ${ }^{18,19}$ that optical defocus can affect colour vision perception, suggesting that significant uncorrected refractive errors can affect colour vision. In the present study, it was found that no abnormality in colour vision perception was observed with VA of $6 / 24$ or better. Higher values of VA may, however, affect colour vision findings. McCulley et al. ${ }^{19}$, however, reported that normal colour perception can be found up to $1.4 \log$ MAR (Snellen acuity of 6/85) for a similar test (Panel D15). There are several factors that may be responsible for this discrepancy such as differences in the VA method employed in the studies. McCulley et al. ${ }^{19}$ used $\log$ MAR chart, whereas, we employed the Snellen acuity chart. Also, it is possible that they used a standard panel D-15, whereas we used the desaturated version, which is more demanding. Other differences in methodology may also contribute to the differences. For example, in our study, optical defocus was done at 6 meters to simulate how distance refractive error such as myopia might affect colour vision findings, while McCulley et al., ${ }^{19}$ did their optical defocous and VA measurement at near, presumably at $40 \mathrm{~cm}$. Another major difference between the present study and that of McCulley et $a l .{ }^{19}$ is the differences in the number of subjects. Their subjects were only 11 whereas, there were 29 subjects in the present study. Considering the wide inter-subject variability in the way that optical defocus affects colour vision, large differences in the number of subjects could significantly influence findings and this may also account for the difference in the visual acuity values reported. Another important source of differences in the quantitative findings between the present study and those of McCulley et al. ${ }^{19}$ is the way the later authors arrived at their visual acuities. They calculated for each test, the average logMAR acuity at which participants had a 10\% reduction from baseline colour vision and subtracted from this, two standard deviations to estimate the acuity at which $5 \%$ of the population should first experience a reduction in colour vision testing. Whereas, we reported the VA that we found experimentally at which failure in colour vision test occurred. This difference in interpretation of results may have contributed to the differences in visual acuity values.

A major concern raised during the conference and by the reviewers of this article was the fact that the VA measurements and optical defocus were done at far rather that at near, while the colour vision testing was done at near. This is a valid concern, but this route was taken because we were interested in how reduced distance VA as in myopia could affect colour perception generally. The only effect that we anticipated that this could have had on the findings was to decrease the positive lens power needed for optical defocus compared with near. This procedure would not affect our conclusion that optical defocus would affect colour vision testing. This claim is supported by the findings of McCulley et al., ${ }^{19}$ who did their optical defocus at near and found that the colour perception was not affected until VA of $1.4 \log$ MAR (Snellen VA of 6/85) for a similar test (Panel D15).

We agree with McCulley et al. ${ }^{19}$, that these optical defocus findings may not apply to subjects with reduced VA attributable to causes other than uncompensated refractive errors resulting in degraded visual acuity. Reduced VA resulting from ocular diseases such as optic atrophy, hypertensive retinopathy or diabetic retinopathy would obviously yield different findings from those of optical defocus.

\section{Conclusion}

Large uncorrected refractive errors resulting in decreased VA worse than $6 / 24$ can affect colour vision findings. Therefore, we recommend that such refractive errors should be neutralized before colour vision testing is embarked upon. This will ensure that refractive errors do not interfere with colour vision findings. The findings here are only applicable to visual acuity degradation resulting from refractive conditions and may not apply to reduced visual acuity from other sources such as ocular diseases. Workers such as train 
drivers whose occupations require keen colour vision must have their vision examined regularly and their refractive errors compensated for to avoid colour misperception which otherwise might result in fatalities.

\section{References}

1. Neitz M and Neitz J. Molecular genetics of colour vision and colour vision defects. Arch Ophthalmol 2000 118 691-700.

2. Vorobyev, M. Ecology and evolution of primate colour vision. Clin Exper Optom 2004 87 230-238.

3. Hardy RA. Retina and intra-ocular tumors. In :General ophthalmology. Vaughan DG, Asbury A, Riordan-Eva P. Norwalk: Prentice Hall International, 1995.

4. Birch J. A practical guide for colour vision examination: Report of the standardization committee of the International Research Group on colour-vision deficiency. Ophthal Physiol Opt 19855 265-285.

5. Iregren, A, Andersson M, Nylen P. Colour vision and occupational chemical exposures: 1. an overview and effects. Neurotox $200223719-$ 733.

6. Rubin A. Fundamentals of colour awareness: A literature review. S Afr Optom 200564 102-109.

7. Institute of Electrical Engineers (IEE). Colour vision defects: A health and safety fact sheet. Herts: Fact sheet No. 2.1, 2004

8. Whillans MG and Allen MJ. Colour defective drivers and safety. Optom Vis Sci 199269 463-466.

9. Vingrys A J and Cole BL. Are colour vision standard justified for the transport industry? Ophthal Physiol Opt 1988 $8259-274$.

10. Haegerstrom-Portnoy G. Colour vision. In: Princiles and practice of pediatric optometry. Rosenbloom AA and Morgan MW. (eds). Philadelphia: JB Lippincott Co. 1990 449-446.

11. Birch J. Diagnosis of defective colour vision. London: Butterworth-Heinemann, 1993, p 51.

12. Good GW, Schepler A, Nichols JJ. The reliability of the Lanthony desaturated D-15 test. Optom Vis Sci 2005 82 $1054-1059$.

13. Press LJ, More BD. Clinical pediatric optometry. Butterworth-Heinemann, Boston, 1993, p 72.

14. Grossvenor T. Primary care optometry, $4^{\text {th }}$ Ed. Butterworth-Heinemann, Boston, 2002.

15. Kocak-Altinas AG, Satana B, Cokak I and Duman S. Visual acuity and colour vision deficiency in amblyopia. Europ J Ophthalmol $2000 \mathbf{1 0}$ 77-81.

16. Thorn F. Effects of dioptric blur on Vistech contrast sensitivity test. Optom Vis Sci 199067 8-12.

17. Rabin J. Optical defocus: Differential effects on the size and contrast letter recognition thresholds. Invest Ophthalmol Vis Sci 1994 35 646648.

18. Wood JM, Atchison DA, Chaparro A. When red lights look yellow. Invest Ophthalmol Vis Sci 2005 45 4338-4252.

19. McCulley TJ, Golnick KC, Lam LL, Feuer MS. The effect of decreased visual acuity on clinical colour testing. Am J Ophthalmol 2006 141 194-196. 